\title{
Exponential Growth and Solvents-Production of Clostridium acetobutylicum ATCC 824 on TYA Media Containing Sucrose and Glucose as Different Sole Carbon Sources
}

\author{
Elizabeth Omolola Oladapo*, Enimie Endurance Oaikhena, Mohammed Sani Abdulsalami \\ Department of Biological Sciences, Nigerian Defence Academy, Kaduna, Nigeria \\ Email address: \\ fatanmielizabeth@yahoo.com (E. O. Oladapo), oaikhenaeni@yahoo.com (E. E. Oaikhena), msabdulsalami@nda.edu.ng (M. S. Abdulsalami) \\ ${ }^{*}$ Corresponding author
}

\section{To cite this article:}

Elizabeth Omolola Oladapo, Enimie Endurance Oaikhena, Mohammed Sani Abdulsalami. Exponential Growth and Solvents-Production of Clostridium acetobutylicum ATCC 824 on TYA Media Containing Sucrose and Glucose as Different Sole Carbon Sources. American Journal of BioScience. Vol. 5, No. 4, 2017, pp. 64-69. doi: 10.11648/j.ajbio.20170504.12

Received: March 26, 2017; Accepted: April 19, 2017; Published: June 19, 2017

\begin{abstract}
Clostridium acetobutylicum ATCC 824 is a solventogenic, obligate anaerobic bacterium that can grow on various types of carbohydrates and are capable of producing spores. In the present study, Clostridium acetobutylicum was successfully grown on TYA medium (tryptone, yeast, acetate medium) containing two different carbon sources, glucose and sucrose coupled with the production of acids (butyric and acetic acid) and solvents, ABE (acetone, butanol and ethanol). An investigation was undertaken to determine the impact of the two types of carbon sources on the solvent production and growth of Clostridium acetobutylicum. HPLC and GC analysis revealed the amount of acids and solvents produced respectively, as well as the amount of unutilized sugars. The amount of combined ABE produced on glucose $(0.19 \mathrm{~g} / 1 \mathrm{~A}, 0.39 \mathrm{~g} / \mathrm{l} \mathrm{B}, 0.06 \mathrm{~g} / \mathrm{E})$ was higher than on sucrose as carbon source $(0.15 \mathrm{~g} / 1 \mathrm{~A}, 0.30 \mathrm{~g} / \mathrm{l} \mathrm{B}, 0.03 \mathrm{~g} / \mathrm{l}$ E). The colony forming units of Clostridium acetobutylicum grown on glucose $\left(4.70 \times 10^{5}\right.$ units $\left./ \mathrm{ml}\right)$ was higher than on sucrose $\left(0.1 \times 10^{5}\right)$ as judged by dilution spread plating on agar. Hence, Glucose was confirmed as the carbon source characterized by the best performance for solvents production and growth of the bacterium. The whole production process on both glucose and sucrose was observed to mainly influence the production of butanol with the concentration of $0.39 \mathrm{~g} / 1$ and $0.30 \mathrm{~g} / 1$ respectively, over the production of other solvents. Higher amount of solvents was produced at lower $\mathrm{pH}$ in both cultures with the different carbon sources. Wet-mounts, gram stain and endospore stain were used to determine the motility, type and sporulation of Clostridium acetobutylicum respectively. Acidogenic phase which seems to couple with the growth of vegetative cells, results into production of acetic and butyric acids. Solventogenic phase commences with a drop in $\mathrm{pH}$ and is accompanied by the onset of sporulation.
\end{abstract}

Keywords: Clostridium acetobutylicum ATCC 824, Sucrose, Glucose, ABE

\section{Introduction}

Clostridium acetobutylicum ATCC 824 is an obligate anaerobe, Gram positive solventogenic bacterium that grows heterotrophically on various carbohydrates such as glucose, cellobiose and xylose. This bacterium is best known for its fermentative pathway which results in production of commercially significant solvents; butanol, acetone, and ethanol [1].

Members of the genus Clostridium include C. difficile, C. pefringens and $\mathrm{C}$. botulinum: these are toxin producing species with much medical significance. Other significant species which are solventogenic species are C. acetobutylicum, C. beijerinckii, C. saccharobutylicum and C. thermocellum [1]. Members of the genus Clostridium produce terminal endospores, which confer a high degree of resistance to heat, desiccation and other environmental challenges, therefore, they can survive harsh environment over long periods of time [2]. The spores germinate once the environmental conditions become favourable for living and 
they also get revived if heat shocked ( $>80$ degree centigrade at 10 minutes) [3]. Sporulation is triggered by low $\mathrm{pH}$, the presence of carbohydrates and at times a quorum sensing mechanism at high population densities $[4,5]$.

The metabolic pathway of the Clostridium acetobutylicum is divided into two distinct phases: acidogenic phase and solventogenic phase [6]. In the first phase, acidogenic phase, the bacterium uses the carbon sources in the media, and transforms them into organic acids (acetic acid and butyric acid) with energy release. In the second phase which is the solventogenic phase, the acids, toxic to the bacterium, are converted into solvents (acetone, butanol and ethanol) [7]; this occurs during the stationary phase in batch cultivation. This process is termed ABE (acetone: butanol: ethanol) process $[8,9]$. It has been reported that production of solvents by the members of the genus Clostridium was induced by specific carbohydrates. Nevertheless, the possibility of using glucose and sucrose has not been explored thoroughly, especially for strains like C. acetobutylicum ATCC 824 [10].

The objective of this study is to investigate the cell growth and solvent production by the bacterium Clostridium acetobutylicum in relation to the different types of carbon sources (glucose and sucrose) used as substrates and also to determine the factors that can affect the switching of phase from acidogenesis to solventogenesis. The information received from this study might be used to better understand acidogenic - solventogenic process and the best carbon source to use for the improvement of growth and solvent production by Clostridium acetobutylicum.

\section{Materials and Methods}

\subsection{Bacterial Strains, Media and Growth Conditions}

Clostridium acetobutylicum ATCC 824 spores stock culture was germinated in Reinforced Clostrida Medium (RCM; Oxoid): The spores $(0.5-1 \mathrm{ml})$ stock was heat shocked in sterile glass tube at $80^{\circ} \mathrm{C}$ for 10 minutes and immediately transferred to $20 \mathrm{ml} \mathrm{RCM}$ in the anaerobic workstation. The stock culture was inoculated overnight. For the production of precultures on TYA (tryptone, yeast, acetate medium) prior to growth experiment, 20ml TYA medium + glucose ( $1 \%$ solution) was inoculated with the overnight culture and was grown overnight. All growth experiments were performed anaerobically in an anaerobic chamber (Mininacs Anaerobic Work Station, Don Whitely) at $37^{\circ} \mathrm{C}$ temperature because Clostridium acetobutylicum is an obligate anaerobe.

For the growth experiments, different carbon sources, glucose and sucrose $(20 \mathrm{ml}$ volumes- $10 \mathrm{~g} / \mathrm{l})$ were added to two shake flask each containing $80 \mathrm{ml}$ TYA and were inoculated with $5 \mathrm{ml}$ of the overnight Clostridium acetobutylicum culture.

For growth analysis, 10-fold Serial dilutions and spread plates of the inoculating culture (day 0 ) and anaerobic test cultures (day 7) were performed as described by [11] using aseptic technique. These plates were examined, colony forming units were determined and $\mathrm{pH}$ measured using a bench top $\mathrm{pH}$ meter (Seven Easy Mettle Toledo, $20.4^{\circ} \mathrm{C}$ ) after a seven-day static incubation in the anaerobic chamber. Equation 1 presents how Colony forming units per millilitre of the original culture was calculated.

$$
C F U m l^{-1}=\frac{A}{D \times V}
$$

Where $\mathrm{CFU}=$ Colony Forming units

$\mathrm{A}=$ Average number of colonies on dilution plate (from replicates)

$\mathrm{D}=$ Dilution plated

$\mathrm{V}=$ volume plated $(\mathrm{ml})$

Samples taken at day 0 were used to gain baseline data on cell density by spectrophotometer (Jenway 6300 spectrophotometer, Optical density at wavelength $\mathrm{OD}_{600}$ ), colony forming units on dilution spread plates (taken from inoculating culture) and medium $\mathrm{pH}$.

\subsection{Sugar and Solvent Analysis}

Samples for each inoculated growth medium were transferred to sterile micro-centrifuge tubes and centrifuged (IEC Micromax centrifuge) at $12000 \mathrm{rpm}$ for 10 minutes. The supernatant was carefully removed. $10 \mu 1$ propan-1-ol was added to $1 \mathrm{ml}$ of the supernatant to determine the solvent concentration using GC (Gas Chromatography) Chrompack 9001. $1.25 \mu \mathrm{l} 1 \mathrm{M} \mathrm{H}_{2} \mathrm{SO}_{4}$ was added to another $1 \mathrm{ml}$ of the supernatant to determine the acids and sugar concentrations using HPLC (High Performance Liquid Chromatography). HPLC and GC analysis were performed by Biofuel Research Centre, Edinburgh Napier University and the data was duly provided.

\subsection{Motility Testing}

To determine whether Clostridium acetobutylicum is motile or not, a sample of wet-mounts of the culture suspension (liquid culture) was examined directly under the bright-field microscope (Leica CMC Classic) using the X40 lens objective and under oil immersion, using the x100 lens objective.

\subsection{Air Dried Smears and Staining}

Air dried smear of the Clostridium acetobutylicum culture was performed as described by [12] for staining. Gram staining as modified by [13] and endospore staining Schaeffer-Fulton's Technique [14] of the smear were performed and observed by the bright-field microscopy. At this point, the vegetative cells, endospores, their colour, shape, position and number were identified and some images were taken while some others were drawn as seen.

The Sampling and analysis as stated above were repeated after 7 days. Growth of Clostridium acetobutylicum, substrate utilisation, changes in cell morphology and staining characteristics, solvent production, yield and ratios on different carbon sources, change in cell motility as well as change in medium $\mathrm{pH}$ were examined and all results recorded. 


\section{Results}

\subsection{Growth and Solvent Production by Clostridium Acetobutylicum on Different Substrates}

The concentrations of solvents (ethanol, acetone and butanol) and acids (acetic and butyric acid) were determined after 7 days of static incubation in the anaerobic chamber. For Day 0 culture, no solvent production was detected from neither glucose nor sucrose fermentation. Nevertheless, on day 7 culture, both substrates were degraded by the bacterium. Substrate utilization was shown to have increased, the value of substrate unused rapidly dropped from $9.87 \mathrm{~g} / 1$ to $2.80 \mathrm{~g} / \mathrm{l}$ (glucose concentration) and $9.57 \mathrm{~g} / 1$ to $3.45 \mathrm{~g} / 1$ (sucrose concentration) (Table 1).

The $\mathrm{pH}$ readings also show decrease in $\mathrm{pH}$ (6.68 to 5.30 for glucose) and the solvent concentration is high at low $\mathrm{pH}$ (Table 1). Optical density measurements for each growth medium (glucose and sucrose media) on day $0,0.241$ (glucose medium) and 0.164 (sucrose medium) increased to 1.86 (glucose) and (sucrose medium) by day 7 (Table 1).

A high level of solvent production was observed during growth on glucose with the amount of butanol as the highest among other solvents produced, followed by acetone and then the least, ethanol (Figure 1). Growth on the agar spread plates for both day 0 inocualting culture and day 7 anaerobic test cultures (containing the two carbon sources) was examined after a 7-day static incubation in the anaerobic chamber and colony forming units were determined. It was observed that Clostridium acetobutylicum grew very well on glucose $(4.70 \times 105$ units $/ \mathrm{ml})$ as carbon source than on sucrose $(0.10 \times 105$ units $/ \mathrm{ml})$ as carbon source (Table 2). The cell count of Clostridium acetobutylicum the inoculating culture medium becomes multiplied in proportion (exponential) over the 7-day period.

ABE (acetone: butanol: ethanol) yield as grams of ABE produced per grams of sugar consumed was determined and was observed to be higher in culture grown on glucose than culture grown on sucrose by the high yield of butanol, 0.06gg-1. ABE fermentation Clostridium acetobutylicum produces acetone, butanol and ethanol in a ratio of $3: 6: 1$ respectively $(0.19 \mathrm{~g} / 1$ acetone, $0.39 \mathrm{~g} / 1$ butanol and $0.06 \mathrm{~g} / \mathrm{l}$ ethanol) with a total glucose concentration of $10 \mathrm{~g} / \mathrm{l}$ and $3: 5: 1$ with sucrose concentration of $10 \mathrm{~g} / \mathrm{l}$ in the original flask (Table 3).

\subsection{Cell Micromorphology, Motility, Gram and Endospore Stains Results}

For each of the day 0 and day 7 culture suspension of Clostridium acetobutylicum, clostridia rods were seen; higher movement of cells was observed in day 7 culture than in day 0 cultures under oil immersion using x100 lens objective with a light microscope.

Purple rod shaped cells were observed when air dried smears of the bacterium culture were stained with crystal violet, counter stained with safranin $O$ (gram stain) and viewed under oil immersion using the x100 lens objective for both day 0 and day 7 cultures. Cells appeared longer and bigger in the day 7 culture than in day 0 .

Endospores stained dark green as observed under light microscope, when the day 7 culture cell suspensions grown on the two carbon sources were stained with malachite green and counter-stained with safranin $O$. One endospore was seen on cell suspension with sucrose and glucose as carbon source. While for the day 0 culture, vegetative cells were observed as they were stained pink; no endospore was seen on day 0 culture.

Table 1. Solvents (acetone, butanol and ethanol) and acids (butyric and acetic acid) produced by Clostridium acetobutylicum on different substrates (glucose or sucrose) at $10 \mathrm{~g} / \mathrm{L}$ solution, their optical density and $\mathrm{pH}$ readings for Day 0 and Day 7 cultures.

\begin{tabular}{|c|c|c|c|c|c|c|c|}
\hline Day 0 & & Amount of product (g/litre) & & & & $\mathrm{OD}_{600 / 650}$ (units) & \\
\hline Substrate (amount unsed) & Acetic acid & Butyric acid & Acetone & Butanol & Ethanol & Optical Density & $\mathrm{pH}$ readings \\
\hline Glucose (9.87) & 0.03 & 0.02 & ND & ND & ND & 0.241 & 6.68 \\
\hline Sucrose (9.57) & ND & $\mathrm{ND}$ & ND & ND & ND & 0.164 & 6.65 \\
\hline Day 7 & & Amount of product (g/litre) & & & & $\mathrm{OD}_{600 / 650}$ (units) & \\
\hline Substrate (amount unused) & Acetic acid & Butyric acid & Acetone & Butanol & Ethanol & Optical Density & $\mathrm{pH}$ reading \\
\hline Glucose (2.80) & 0.01 & 0.01 & 0.19 & 0.39 & 0.06 & 1.86 & 5.30 \\
\hline Sucrose (3.45) & ND & ND & 0.15 & 0.30 & 0.03 & 1.89 & 5.63 \\
\hline
\end{tabular}

ND: Not Determined

Table 2. Colony forming units of Clostridium acetobutylicum on dilution plates taken from inoculating culture (day 0) and anaerobic test cultures, on the two carbon source (day 7$)$.

\begin{tabular}{ll}
\hline Day $\mathbf{0}$ & Colony Forming Units (units/ml) \\
\hline Inoculating culture medium & $0.02 \times 10^{5}$ \\
Day 7 & \\
Culture with glucose as carbon source & $4.70 \times 10^{5}$ \\
Culture with sucrose as carbon source & $0.10 \times 10^{5}$ \\
\hline
\end{tabular}




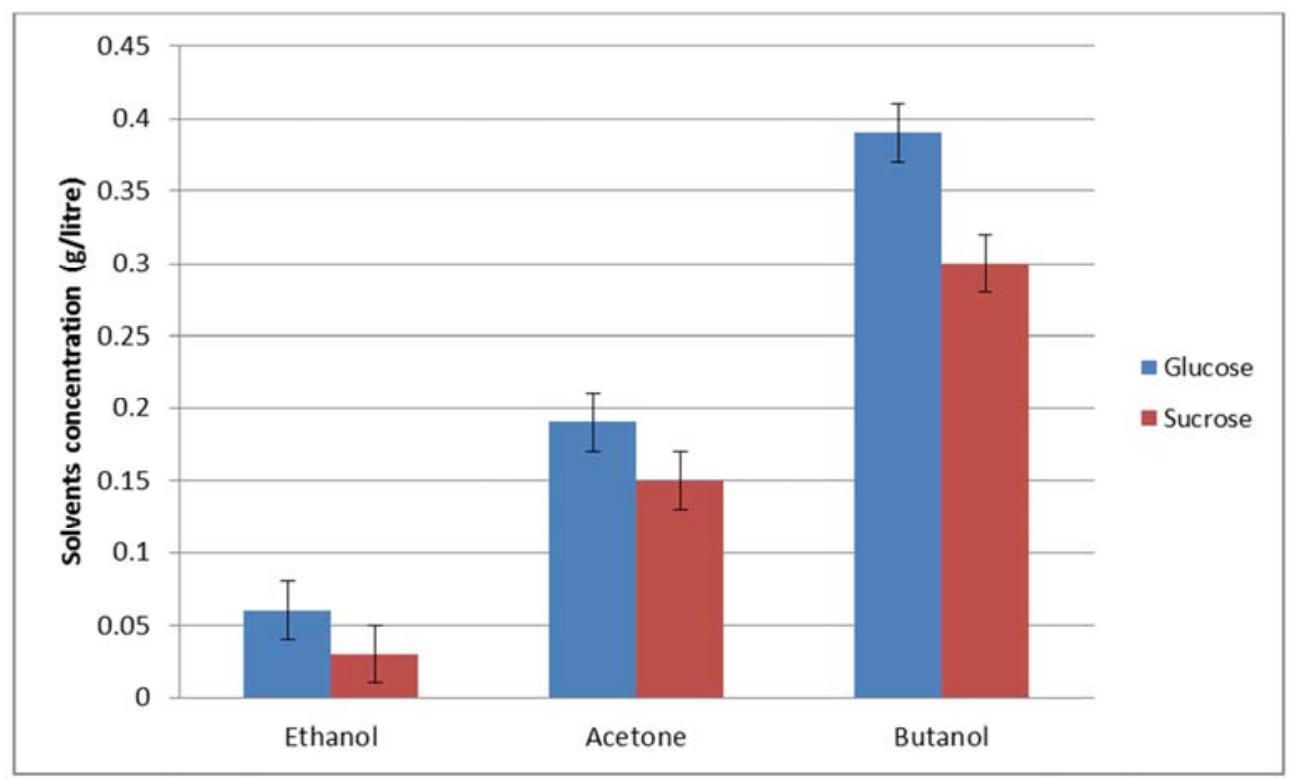

Figure 1. Solvents produced by Clostridium acetobutylicum on substrates with different carbon sources. Bar with blue shade indicates substrate with glucose as carbon source. Red bar represent substrate with sucrose as carbon source.

Table 3. Acetone Butanol Ethanol yield from $10 \mathrm{~g}$ of substrate (glucose and sucrose) per Litre in the fermentation reactor of Clostridium acetobutylicum. ABE ratio also determined and stated.

\begin{tabular}{lllllll}
\hline & & Glucose as carbon source & & \multicolumn{2}{l}{ Sucrose as carbon source } \\
\hline & Acetone & Butanol & Ethanol & Acetone & Butanol & Ethanol \\
\hline Amount of Solvents formed $(\mathrm{g} / \mathrm{l})$ & 0.19 & 0.39 & 0.06 & 0.15 & 0.30 & 0.03 \\
Substrate used $(\mathrm{g} / \mathrm{l})$ & 7.2 & 7.2 & 7.2 & 6.5 & 6.5 & 6.5 \\
Solvent yield gg & 0.03 & 0.06 & 0.01 & 0.02 & 0.05 & 0.01 \\
ABE ratio & 3 & 6 & 1 & 3 & 5 & 1 \\
\hline
\end{tabular}

\section{Discussions}

Clostridium acetobutylicum ATCC 824 utilizes both glucose and sucrose for growth and solvent production. This experiment shows that the profitability of production of solvents and fermentation is dependent on the type and concentration of carbon sources used as substrates, the $\mathrm{pH}$, culture medium design, optical density and product yield.

Two significant parameters that affected the production of solvents and growth of the bacterium are the $\mathrm{pH}$ condition and acid concentrations. According to the day 0 result (Table 1), there was no production of solvent, indicating acidogenic phase. Day 7 indicates solventogenic phase. Initially, $\mathrm{pH}$ was at 6.68 for acidogenesis (day 0 ), then a change to $\mathrm{pH} 5.30$ induced a metabolic shift to solvent production phase. Thus, implies that decrease in $\mathrm{pH}$ is a requirement for solventogenesis. This is in line with the research done by [15], where it was reported that increase in butyric acid concentration and decrease in $\mathrm{pH}$ are usually needed for the initiation of a metabolic shift from acidogenesis to solventogenesis. Certain levels of acids concentrations (butyric and acetic acids) are necessary for the initiation of solvents production [16]; this correlates with this experiment where solventogenesis was induced on day 7 , when the concentrations of acids had reached a certain level $(0.04 \mathrm{~g} / 1$ and $0.05 \mathrm{~g} / \mathrm{l}$ ) from $0.03 \mathrm{~g} / \mathrm{l}$ and $0.02 \mathrm{~g} / \mathrm{l}$ for acetic and butyric acids respectively. In the first phase, acidogenic phase, the bacterium utilizes the carbon sources in the media, and transforms them into organic acids (acetic acid and butyric acid) with the release of energy [17]. During acidogenesis (day 0 ), the bacterium dominantly produces acids, whereas the solvents; acetone, butanol and ethanol are the major fermentation products at solventogenesis (day 7). Acid production leads to a decrease in $\mathrm{pH}$, hence, confers stress on the bacterium [18]. Nevertheless, as cells enter stationary phase, in response to cellular and environmental factors, the bacterium performs a metabolic switch, where acidogenesis is ends and the acids formed during acidogenesis are utilized to convert them into organic solvents (butanol, acetone, and ethanol) [19]. It can be deduced that Clostridium acetobutylicum changes its metabolism between acidogenic phase and solventogenic phase in response to changes in the $\mathrm{pH}$. Thus, production of solvents is usually associated with the uptake of acids.

The solvents yield from glucose was higher than that of sucrose (Figure 1). Despite the use of different types of substrates in this experiment the yield of combined $\mathrm{ABE}$ (Acetone: Butanol: Ethanol) never exceeded $0.10 \mathrm{gg}^{-1}$ (Table 3)' this may be due to the fact that there was incomplete sugar utilization that is $2.8 \mathrm{~g} / 1$ unutilized glucose (Table 1 ) because the HPLC and GC analysis were carried out on contents from small bottles and may also be that the solventogenic phase just began and had no enough time yet to utilize well the 
substrate as there are still unused substrate and have not yet re-assimilated all the acids produced in the acidogenic phase. Though, according to research, even with the use of cell recycle, immobilization of cell extractive fermentation for cell density and productivity increment, the yield of $\mathrm{ABE}$ production was never beyond $0.44 \mathrm{gg}^{-1}$ [20]. Nevertheless, substrate with glucose as carbon source yielded the combined ABE solvent more than sucrose with butanol as the main solvent produced. It can thus be deduced that the whole fermentation process and condition favours the production of butanol over other solvents (Figure 1).

Based upon the results of this experiment, the bacterium, Clostridium acetobutylicum are gram positive bacterium because they stained purple with gram stain. They have rodlike shape. Vegetative cells that were observed on day 0 , in relation with the fact that only acidogenic phase was in place for day 0 implies that acidogenesis was coupled with the growth of vegetative cells. Acidogenic phase coupled with vegetative cell growth, forms acetic and butyric acids with gas (such as carbohydrate and hydrogen) as the main products [21].

Sporulation started to occur on day 7 , whereas solventogenic phase also began on day 7 , indicating that solventogenesis is likely to be accompanied by the onset of sporulation.

Due to the changes in the morphology that is, longer cells, higher motility, spore formation, in the bacterium, one could possibly differentiate the acidogenesis from the solventogenesis in a population of spore forming cells. Cells are usually highly motile and quickly dividing after spore germination. In addition, cell swelling is usually followed by pre-spore formation [22]. The higher colony forming units of the bacterium grown on the different carbon sources as observed on the agar spread plates on day 7 than on day 0 was probably due to the cells quickly dividing over the period of 7 days. It can therefore be said that the exponential growth of the bacterium over the 7-day period $\left(4.7 \times 10^{5}\right.$ units $/ \mathrm{ml}$ and $0,1 \times 10^{5}$ unit $/ \mathrm{ml}$ for glucose and sucrose respectively), which is far more than twice as numerous as the day 0 innoculating culture $\left(0.02 \times 10^{5}\right.$ unit $\left./ \mathrm{ml}\right)$ is thus characterized by the production of acids (acidogenesis). Under normal conditions, $C$. acetobutylicum enters an exponential growth phase which is correlated to the formation of acids, acetate and butyrate as predominant liquid fermentation products [23].

Optical density values for both day 0 and day 7 indicate the concentration of bacteria in both suspensions with sucrose and glucose as carbon sources. Greater OD units (1.86 $\mathrm{OD}_{600}$ units) in day 7 cell suspension indicate that more bacteria and much solvent are present. This, therefore confirms the production of solvents on day 7 .

\section{Conclusions}

The results obtained from fermentation of different carbon sources (glucose and sucrose) showed that $C$. acetobutylicum is capable of utilizing products of biomass containing these sugars for the production of solvents (ABE). Glucose was confirmed as the carbon source characterized by the best performance for solvent production and growth of $C$. acetobutylicum. C. acetobutylicum prefers glucose as source of carbon and energy [20]. The fermentation performances of glucose can thus, be further researched and optimized. Acidogenic phase in relation to vegetative cell growth, results into production of acetic and butyric acids. Solventogenic phase commences with a drop in $\mathrm{pH}$ and is accompanied by the beginning of sporulation, re-assimilating some of the acids formed into producing acetone, ethanol and mainly butanol. Clostridium acetobutylicum seems to have a greater potential for efficient butanol production with the use of glucose than of sucrose as substrate. Nevertheless, if this process can be optimized to produce solely butanol, it would be an attractive process for the production of butanol for alternative biofuel.

A process that will reduce acidogenesis phase and promote early solventogenesis should also be investigated for future purpose. Moreover, the strict anaerobic feature of $C$. acetobutylicum and its preferential feature to sugars as carbon source hinder future applications [23]. Hence, a further research on $C$. acetobutylicum metabolism and its regulations is necessary for future industrial-scale applications.

\section{References}

[1] Bankar, S. B., Survase, S. A., Singhal, R. S. and Granstrom, T. (2011). Continuous two stage acetone-butanol-ethanol fermentation with integrated solvent removal using Clostridium acetobutylicum B 5313. Bioresource Technology. 106: 110-116.

[2] Lynd, L. R., Weimer, P. J. and Zyl, W. H. (2002). Pretorius IS: Microbial cellulose utilization: fundamentals and biotechnology. Microbiology and Molecular Biology Reviews. 66(3): 506-577.

[3] Barer, M. R. (2003). Physiological and molecular aspects of growth, non-growth, culturability and viability in bacteria. Cambridge University Press, Cambridge.

[4] Li, J., Chen, J., Vidal, J. E. and McClane, B. A. (2011). The Agar-like quorum-sensing system regulates sporulation and production of enterotoxin and beta2 toxin by Clostridium perfringens type A non-food-borne human gastrointestinal disease strain F5603. Infection and Immunity. 79(6): 24512459 .

[5] Sandoval, N., Venkataramanan, K., Groth, T. and Papoutsakis, E. (2015). Whole-genome sequence of an evolved Clostridium pasteurianum strain reveals Spo0A deficiency responsible for increased butanol production and superior growth. Biotechnology for Biofuels. 8: 1.

[6] Lutke-Eversloh, T. and Bahl, H. (2011). Metabolic engineering of Clostridium acetobutylicum: recent advances to improve butanol production. Current Opinion in Biotechnology. 22: 1-14.

[7] Monot, F., Martin, J. R., Petitdemange, H. and Gay, R. (1982). Acetone and butanol production by Clostridium acetobutylicum in a synthetic medium. Applied and Environmental Microbiology. 44: 1318-1324. 
[8] Gehin, A., Gelhaye, E., Raval, G. and Petitdemange, H. (1995). Clostridium cellulolyticum Viability and Sporulation under Cellobiose Starvation Conditions. Applied and Environmental Microbiology. 61(3): 868-871.

[9] Sarchami, E., Johnson, L. and Rehmann (2016). Optimization of fermentation condition favoring butanol production from glycerol by Clostridium pasteurianum DSM 525. Bioresource Technology 208: 73-80.

[10] Servinsky, M. D., Kiel, J. T., Dupuy, N. F. and Sund, C. J. (2010). Transcriptomal analysis of differential carbohydrate utilization by Clostridium acetobutylicum. Microbiology. 156: 3478-3491.

[11] Sreekumar, S., Baer, Z. C., Gross E. and Padmanaban (2014). S. chemo catalytic upgrading of tailored fermentation product toward biodiesel. Chem. Sus. Chem. 7: 2445 - 2448

[12] Lowenthai, R. M. and Marsden, K. A. (1986). A rapid, simple method for Leukemia immunophenotyping using air-dried blood \& bone marrow sensors. Journal of Immunological Methods. 93: 19-27.

[13] Hucker, G. J. and Conn, H. J. (1923). Method of Gram Staining. New York State Agricultural Experiment Station Technical Bulletin. 93: 1-20.

[14] Harley, J. P. and Prescott, L. M. (2002). Laboratory Exercises in Microbiology. 5th Edition, McGraw Hill, New York.

[15] Gottschal, J. and Morris, J. C. (1981). Non-production of acetone and butanol by Clostridium acetobutylicum during glucose and ammonium-limitation in continuous culture. Biotechnology Letters. 3: 525-530.
[16] Terracciano, J. S. and Kashket, E. R. (1986). Intracellular conditions required for initiation of solvent production by Clostridium acetobutylicum. Applied Environmental Microbiology. 52: 86-91.

[17] Borman, S. (2014). Engineering Clostridium acetobutylicum for production of kerosene and biodiesel blendstock precursors. Metabolic Engineering. 25: 124-130.

[18] Jones, D. T. and Woods, D. R. (1986). Acetone-butanol fermentation revisited. Microbiology Reviews. 50: 484-524.

[19] Ehsaan, M., Kuit, W., Zhang, Y., Cartman, S., Heap, J., Winzer, K. and Minton, P. (2016). Mutant generation by allelic exchange and genome resequencing of the biobutanol organism Clostridium acetobutylicum ATCC 824. Biotechnology of Biofuels. 9: 4.

[20] Mitchell, W. J. (1998). Physiology of carbohydrate to solvent conversion by clostridia. Advanced Microbial Physiology. 39: 31-130.

[21] Kharkwal, S., Karimi, I. A., Chang, M. W. and Lee, D-Y (2009). Strain improvement and process development for biobutanol production. Recent Patents on Biotechnology. 3: 202-210.

[22] Paredes, C. J., Alsaker, K. V. and Papoutsakis, E. T. (2005). A comparative genomic view of clostridial sporulation and physiology. Nature Reviews Microbiology. 3: 969-978.

[23] Millat, T., Holger, J., Hubert, B., Ralf-Jorg, F. and Olaf, W. (2013). The $\mathrm{pH}$-induced metabolic shift from acidogenesis to solventogenesis in Clostridium acetobutylicum. Experimental Standard Conditions of Enzyme Characterization. 1: 33-55. 